\title{
Mechanical Thrombectomy for Acute Ischemic Stroke in Nepal
}

\author{
Pravesh Rajbhandari ${ }^{1}$, Saujanya Rajbhandari ${ }^{2}$, Anish Neupane ${ }^{3}$,Pritam Gurung ${ }^{1}$ \\ ${ }^{1}$ Department of Neurosurgery, Annapurna Neurological Institute and Allied Sciences, Maitighar, Kathmandu, Nepal \\ ${ }^{2}$ Department of Neurosurgery, Hyogo Medical College, Hyogo, Japan \\ ${ }^{3}$ Department of Radiology, Annapurna Neurological Institute and Allied Sciences, Maitighar, Kathmandu, Nepal
}

\section{CORRESPONDENCE}

Dr.Pravesh Rajbhandari, Department of Neurosurgery, Annapurna Neurological Institute and Allied Sciences,

Maitighar, Kathmandu, Nepal

Email: praveshreema@gmail.com

\section{ARTICLE INFO}

Article History

Submitted: 03 November, 2020

Accepted: 19 December, 2020

Published: 8 February,2021

Source of support: None

Conflict of Interest: None

Copyright : (CThe Author(S) 2020

This is an open access article under the Creative Common Attribution license CC-BY 4.0

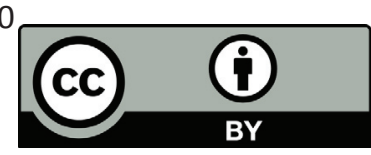

\begin{abstract}
Stroke is one of the leading causes of mortality and the number one cause of dependency in the world. Endovascular therapy has emerged as promising treatment strategy in the patient with acute ischemic stroke due to large vessel occlusion in anterior circulation. Moreover, the time window for mechanical thrombectomy have also been expanded based on the recent DAWN and DEFUSE-3 trial. It is evident that this trend could dramatically increase the number of potential patients for the treatment. Moreover, advancement in stroke imaging have guided physicians to make wisest decision in identifying suitable patient who can get benefit from the recent treatment strategies. The stroke management is evolving and continues to improve, making better outcome of the patient possible. In context of our country Nepal, it is also necessary to educate and aware medical staffs including physicians, nurses, laboratory/ radiology personnel to design a proper acute stroke team to deliver successful therapy which will eventually make a sound impact in a large number of patients with stroke.
\end{abstract}

Herein, we report a case of a 69-year-old gentleman who presented with right middle cerebral artery territory infarction within 3hours of onset of symptoms and underwent mechanical thrombectomy as a part of the treatment.

Keywords: Angiography ; Middle Cerebral Artery (MCA); Mechanical thrombectomy; Thrombolysis in cerebral infarction (TICI).

\section{INTRODUCTION}

Stroke is one of the leading causes of mortality and the number one cause of dependency in the world. Intravenous thrombolysis (IVT) was considered as the most important therapy for the patient with acute ischemic stroke. However, due to its narrow time window, low efficacy and numerous contraindications, IVT was limited to only a certain number of patients.

Lately, several clinical trials showed a clear benefit of endovascular therapy in the patient with acute ischemic stroke due to large vessel occlusion in anterior circulation. Endovascular treatment with stent retriever is recommended up to $8 \mathrm{hrs}$ after symptom onset. However, the time window for the favourable outcome is not well defined which is shown by the recent trials in extending the time window. It is necessary to educate and aware medical staffs including physicians, nurses, laboratory/ radiology personnel in order to design a proper acute stroke team to deliver successful therapy which will eventually make a sound impact in a large number of patients with stroke.

\section{Case Report}

A 69-year-old gentleman presented with sudden onset of left-sided weakness and disorientation since $3 \mathrm{hrs}$ with NIHSS of 6 . He gave history of Hypertension and Atrial Fibrillation under medication warfarin, metoprolol and statin. CT- Scan head done in the previous hospital didn't show any abnormal findings. (Figure 1) DWI showed a hyperintense region in the right middle cerebral artery territory (MCA) with DWI ASPECTS of 8. (Figure 2)

The patient then immediately underwent digital subtraction angiography which confirmed complete occlusion of right MCA at the distal M1 segment. After obtaining consent from the patient's family Mechanical Thrombectomy. After obtaining consent from patient's 


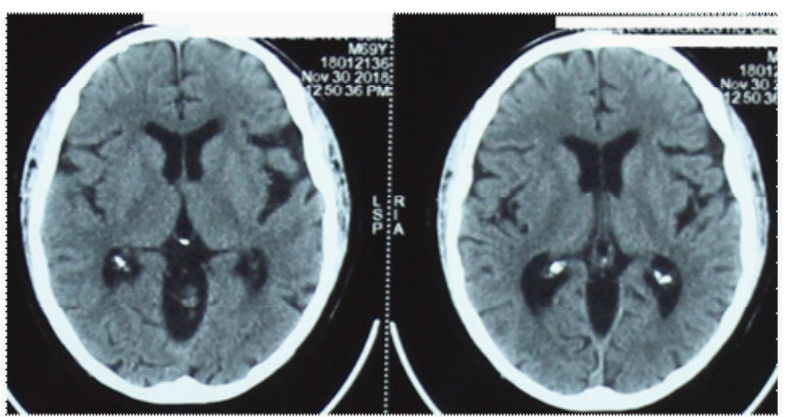

Figure 1. CT-Head from previous hospital showing no signs of abnormality or hemorrhage.

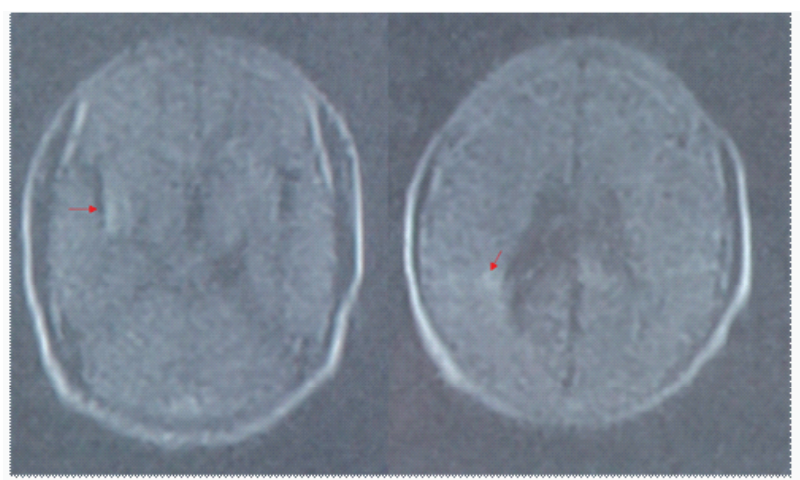

Figure 2: DWI showing hyperintense region (arrows) in right MCA territory with ASPECT score 8.

family ,Mechanical Thrombectomy was performed using stent retriever. A $7 \mathrm{fr}$ long sheath was inserted through the femoral artery under the guidance of the guiding catheter and guidewire. This sheath was placed on the petrous portion of ICA. Microcatheter (VASCO) and micro guidewire passed through the occluded part of the distal M1 to the superior trunk of MCA. Microcatheter was slowly pulled back to deploy pREset thrombectomy retriever 4-20 (phenox, Bochum, Germany). After waiting for $5 \mathrm{~min}$ stent retriever was then gradually pulled back along with microcatheter. (Figure 3) Post-procedure angiography revealed Thrombolysis in cerebral infarction ( $\mathrm{TICl})$ IIB recanalization in first attempt and Distal MCA (M4, M5) occlusion.Distal MCA was not treated due to the risk of haemorrhage.

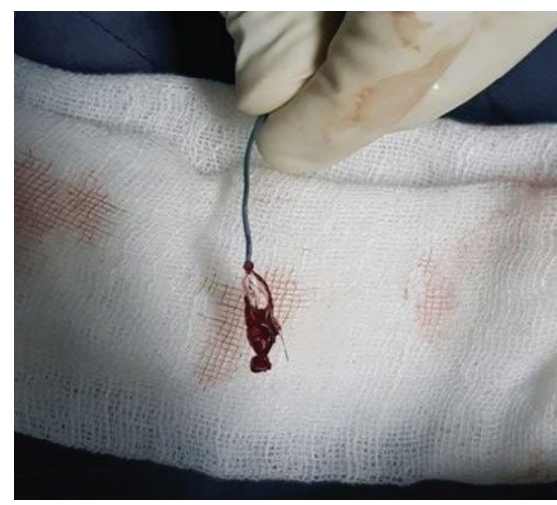

Figure 3: Thrombus in distal M1 removed by preset thrombectomy device
NIHSS did not change after the intervention however it improved from 6 to 2 on the other day with mild weakness of left upper and lower limb. CT- Scan 24 hour after intervention showed right MCA territory infarction. Patient medication was continued, and physiotherapy was encouraged. Patient completely recovered 1 week after the onset of symptoms with Modified Rankin Score (mRS) of 0 .

\section{DISCUSSION}

Stroke is one of the leading causes of mortality and the number one cause of dependency in the world. However, current progress in endovascular therapy has opened a new gateway for the management of acute ischemic stroke. The number of patients eligible for mechanical thrombectomy is increasing with a simultaneous rise in improved outcome and better devices.

A series of trial conducted in different part of the world has shown the effectiveness of mechanical thrombectomy with stent retriever in restoring the flow of occluded vessels including the improvement in functional outcome and the decrease in mortality of the patients.The metaanalysis conducted by HERMES collaborators concluded that endovascular mechanical thrombectomy led to significantly reduced disability compared with standard treatment at 90 days (mRS $0-2,46 \%$ for the interventional arm, and $26.5 \%$ for control population) if performed within $7.3 \mathrm{~h}$ from symptom onset. ${ }^{1}$ Therefore, Mechanical Thrombectomy is recognized as the gold standard of care for patient with large vessel occlusion in anterior circulation. $^{2}$

Moreover, recent trials like DAWN (DWIorCTPAssessment with Clinical Mismatch in the Triage of Wake-Up and Late Presenting Strokes Undergoing Neurointervention with Trevo)and DEFUSE-3 (The Endovascular Therapy Following Imaging Evaluation for Ischemic Stroke)showed that the time window for treatment can be extended up to $24 \mathrm{~h}$ for the patient with anterior circulation strokes keeping in mind the clinical- infarct mismatch. ${ }^{3}$ (Figure 4).

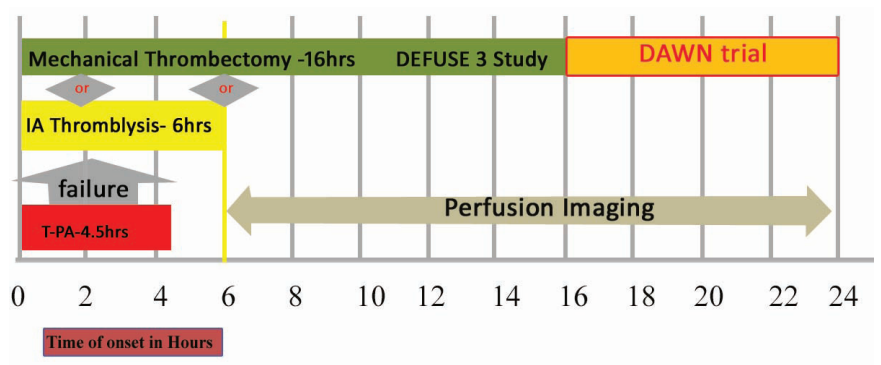

Figure 4: Extended time window for treatment of acute stroke 
Patients with mild or rapidly improving symptoms, but proximal vessel occlusions have a poor outcome when left untreated. Rapid recognition and treatment of such patients are of extremely important. ${ }^{4}$

The patient with large proximal clots primarily located in intracranial ICA and proximal MCA i.e. M1 segment are suitable candidates to achieve benefit from the therapy. However, intervention in at least as distal as the M2 divisions of the MCA can be performed. ${ }^{5}$ Patient with small infarct core with large territories of surrounding hypoxic tissue at the risk of dying are the optimum patient to be treated with mechanical thrombectomy. Advanced imaging and scoring system such as ASPECT should be utilized to recognise such patient in order to preserve the area in the verge of dying.

HERMES collaborators showed thrombectomy without GA is considered to be significantly associated with better outcome. However further study is needed to show the benefits of GA over conscious sedation. ${ }^{4}$ Commonly used thrombectomy techniques are Stent retrievers and direct aspiration at the base of the clot with large bore catheters. Combined technique using the interaction of stent retrievers, aspiration catheter, and position of stent retriever and aspiration catheter in relation to clot are developed to increase the first pass reperfusion rate. ${ }^{5,6}$

Comprehensive stroke centre which has the capability to provide endovascular treatment and Primary stroke centres which provide non-endovascular services including IV tPA should be established. This helps the eligible patient to be referred in an appropriate centre for rapid evaluation and treatment of the eligible patient. ${ }^{7}$ It is also important to educate medical staffs including physicians, nurses, laboratory/ radiology personnel in order to design a proper acute stroke team which will eventually make a sound impact in a large number of patients with stroke.

\section{CONCLUSION}

Mechanical Thrombectomy is considered as the gold standard of care for the patient with large vessel occlusion in the anterior circulation. Proper patient selection and stroke team mobilization is required for achieving successful recanalization.

\section{REFERENCES}

1. Goyal $\mathrm{M}$ et al.Endovascular thrombectomy after largevessel ischaemic stroke: a meta-analysis of individual patient data from five randomised trials. Lancet.2016; 387:1723-31.

2. N. Wahlgren et al.Mechanical thrombectomy in acute ischemic stroke, International Journal of Stroke.2016; vol.11, no. 1, pp. 134-147.

3. Nogueira RG, J. A. (2018). Thrombectomy 6 to 24 hours after stroke with a mismatch between deficit and infarct. N Engl J Med, 378:11-21.

4. Bhogal P et al. Mechanical thrombectomy in patients with M1 occlusion and NIHSS score less than or equal to 5: a single-centre experience. Stroke Vasc Neurol.2016; 1:16571.

5. Sarraj A et al. Endovascular therapy for acute ischemic stroke with occlusion of the middle cerebral artery $\mathrm{M} 2$ segment. JAMA Neurol.2016;73:1291-6.

6. Lapergue B et al. A direct aspiration, first pass technique (ADAPT) versus stent retrievers for acute stroke therapy: an observational comparative study. AJNR Am J Neuroradiol.201637:1860-5.

7. Southerland AM et al. Suspected large vessel occlusion: should emergency medical services transport to the nearest Primary Stroke Center or bypass to a Comprehensive Stroke Center with endovascular capabilities? Stroke.2016; 47:1965-7. 